\title{
Fibrilación auricular en cirugía cardíaca
}

\author{
Atrial fibrillation in cardiac surgery \\ Luis A. Baeza-Herrera1, Gustavo Rojas-Velasco ${ }^{2 *}$, Manlio F. Márquez-Murillo³, Alejandra del R. Portillo-Romero', \\ Lourdes Medina-Paz', Rolando Álvarez-Álvarez², Ángel Ramos-Enríquez y Francisco M. Baranda-Tovar ${ }^{2}$ \\ ${ }^{1}$ Cardiología Clínica; ${ }^{2}$ Departamento de Terapia Intensiva Posquirúrgica; ${ }^{3}$ Departamento de Electrofisiología. Instituto Nacional de Cardiología \\ Ignacio Chávez, Ciudad de México, México
}

\begin{abstract}
Resumen
La fibrilación auricular es la arritmia más frecuente en el periodo posquirúrgico de la cirugía cardíaca. Se relaciona con insuficiencia cardíaca, insuficiencia renal, embolismo sistémico y más días de estancia y mortalidad. La fibrilación auricular en el periodo posquirúrgico de la cirugía cardíaca (FAPCC) suele aparecer en las primeras 48 horas. Los principales mecanismos que producen la aparición y el mantenimiento de la FAPCC son el aumento del tono simpático y la respuesta inflamatoria. Los factores de riesgo adjuntos son la edad avanzada, enfermedad pulmonar obstructiva crónica, enfermedad renal crónica, cirugía valvular, fracción de expulsión del ventrículo izquierdo menor de $40 \%$ e interrupción de fármacos bloqueadores $\beta$. Existen instrumentos que han demostrado predecir la aparición de FAPCC. El tratamiento profiláctico con bloqueadores $\beta$ y amiodarona se relaciona con disminución de la aparición de FAPCC. Dada su naturaleza transitoria, se sugiere que el tratamiento inicial de FAPCC sea el control de la frecuencia cardíaca y sólo en caso de que el tratamiento no consiga el retorno al ritmo sinusal está indicada la cardioversión eléctrica. Se desconoce cuál debe ser el seguimiento a largo plazo y sólo se conocen en escasa medida las complicaciones más allá de este periodo. La FAPCC no es una arritmia benigna ni aislada en los pacientes sometidos a operación cardíaca, por lo que la identificación de los factores de riesgo, su prevención y el seguimiento en el ámbito ambulatorio deben formar parte de las unidades dedicadas a la atención y los cuidados de estos pacientes.
\end{abstract}

Palabras clave: Fibrilación auricular. Cirugía cardíaca. Arritmias en cirugía cardíaca.

\begin{abstract}
Atrial fibrillation is the most frequent arrhythmia in the postoperative period of cardiac surgery. It is associated with heart failure, renal insufficiency, systemic embolism and increase in days of in-hospital and mortality. Atrial fibrillation in the postoperative period of cardiac surgery (FAPCC) usually appears in the first $48 \mathrm{~h}$ after surgery. The main mechanisms involved in the appearance and maintenance of FAPCC are the increase in sympathetic tone and the inflammatory response. The associated risk factors are advanced age, chronic obstructive pulmonary disease, chronic kidney disease, valve surgery, fraction of ejection of the left ventricle $<40 \%$ and the withdrawal of beta-blocker drugs. There are instruments that have been shown
\end{abstract}

\section{Correspondencia:}

*Gustavo Rojas-Velasco

E-mail: gustavorojas08@gmail.com
Disponible en internet: 09-12-2019 Arch Cardiol Mex. 2019;89(4):348-359 www.archivoscardiologia.com BY-NC-ND (http://creativecommons.org/licenses/by-nc-nd/4.0/). 
to predict the appearance of FAPCC. Prophylactic treatment with beta-blockers and amiodarone, is associated with a decrease in the appearance of FAPCC. Given its transient nature, it is suggested that the initial treatment of FAPCC be the heart rate control and only if the treatment does not achieve a return to sinus rhythm, the use of electrical cardioversion is suggested. It is unknown what should be the long-term follow-up and complications beyond this period are little known. FAPCC is not a benign or isolated arrhythmia in patients undergoing cardiac surgery, so the identification of risk factors, their prevention, and follow-up in the outpatient setting, should be part of the units dedicated to the care and care of these patients.

Key words: Atrial fibrillation. Cardiac surgery. Cardiac surgery arrhythmias.

\section{Introducción}

La fibrilación auricular (FA) se puede presentar antes, durante o después de cualquier operación. La FA que surge tras el acto quirúrgico y durante la estancia intrahospitalaria posterior a dicho episodio se denomina FA posquirúrgica. Ésta puede presentarse después de una intervención cardíaca, torácica o no cardíaca. Ésta es una revisión de la FA que aparece luego de un procedimiento cardíaco y en adelante se empleará su sigla FAPCC.

La aparición de la FAPCC conlleva la pérdida de la contribución de la sístole auricular al gasto cardíaco y la disminución del tiempo de llenado diastólico, lo cual puede producir insuficiencia cardíaca o isquemia miocárdica, incremento de los días de ventilación mecánica $(18.2 \%)$, desarrollo de insuficiencia renal (8.5\%) y posibilidad de embolia sistémica. Por consiguiente, la FAPCC se relaciona con un aumento de los días de estancia intrahospitalaria, los costos de atención ${ }^{1}$ y la tasa de mortalidad ${ }^{2}$. De aquí la importancia de intentar prevenir su aparición. En este artículo se revisan los factores de riesgo relacionados con FAPCC y las medidas que se han evaluado para prevenirla con un enfoque basado en la fisiopatología conocida.

\section{Consideraciones clínicas}

La prevalencia de FAPCC varía entre 15 y $40 \%$ en los procedimientos quirúrgicos de revascularización coronaria, 37 a $60 \%$ en los de cirugía valvular y más del $60 \%$ en las intervenciones combinadas y $24 \%$ en los pacientes sometidos a trasplante cardíaco ${ }^{3,4}$. A pesar del avance en el conocimiento de la FAPCC, la incidencia se ha mantenido estable en los últimos 10 años ${ }^{5}$.

El $90 \%$ de los casos de FAPCC se presenta en los primeros cuatro días del periodo posoperatorio ${ }^{3,5}$. La duración promedio del primer episodio es de 7 a 8 horas $^{5}$. La recurrencia ocurre en el $40 \%$ de los casos y se presenta en las primeras 24 horas. Hasta el $80 \%$ de los pacientes se mantiene en ritmo sinusal a las 24 horas tras el primer episodio ${ }^{4}$. El $14 \%$ de los pacientes con FAPCC se mantiene con dicho trastorno rítmico hasta por dos semanas. Los informes de la persistencia de la fibrilación auricular en el seguimiento a largo plazo (3-12 meses) varían, ya que no existe uniformidad en las recomendaciones de seguimiento y en algunos casos no se realiza.

\section{Fisiopatología}

La FAPCC se relaciona con factores, situaciones 0 alteraciones que al final actúan sobre dos mecanismos fisiopatogénicos que son la activación del sistema simpático (aumento de la adrenalina) y la inflamación. Aunque no se conoce el grado de inflamación o el tono simpático necesario para desencadenar la FAPCC, la mayor parte de las medidas dirigidas a su prevención se enfoca en antagonizar a alguno de estos dos efectos ${ }^{6}$.

\section{Fenómeno inflamatorio}

La respuesta inflamatoria en la operación cardíaca puede ser sistémica o local (a nivel del miocardio) y es efecto de la circulación extracorpórea y la cardioplejía 7,8 . La manifestación molecular de la inflamación vinculada con la intervención cardíaca se debe a las citocinas, como las interleucinas 1 y 6 y el factor de necrosis tumoral $\alpha$, entre otras. En el plano cardiovascular, estas citocinas pueden afectar la función contráctil (inotropismo) pero también influir en el remodelado ventricular y el aturdimiento miocárdico, cuya consecuencia es la alteración de los potenciales de acción y el desencadenamiento de arritmias. Además de las citocinas, las especies reactivas de oxígeno (ROS, reactive oxygen species) se vinculan con alteraciones en el potencial de acción del tejido auricular, secundarias a modificaciones en el metabolismo del calcio. En las orejuelas se han descrito cifras elevadas de nicotinamida adenina dinucleótido fosfato como un factor predisponente a la aparición de FAPCC ${ }^{8}$. En modelos animales de FAPCC se ha documentado que la inflamación que aparece alrededor de la cicatriz de 
auriculotomía, expresada por la actividad de la mieloperoxidasa leucocitaria, está directamente relacionada con una mayor dispersión de la activación auricular y con la consiguiente aparición de FA. Además, el tratamiento antiinflamatorio redujo estas alteraciones ${ }^{7}$. Estudios recientes sugieren que la acumulación de sangre dentro del pericardio se vincula con la aparición de FAPCC, efecto también mediado por citocinas y ROS producidas por el metabolismo de los componentes celulares de la sangre y el plasma ${ }^{9}$.

\section{Activación simpática}

La activación neural es otro de los factores que intervienen en el desarrollo de FAPCC. El incremento del tono adrenérgico produce alteraciones en el periodo refractario auricular, aumenta la frecuencia cardíaca y disminuye la variabilidad de los intervalos $R-R$ o la variabilidad de la frecuencia cardíaca, factores que promueven el desarrollo de un sustrato eléctrico para la aparición de FAPCC ${ }^{10}$. De ahí la utilidad de los bloqueadores $\beta$ (BB) en el tratamiento profiláctico.

\section{Factores de riesgo e instrumentos para predecir la aparición de FAPCC}

El principal factor de riesgo relacionado con FAPCC es la edad; por cada incremento de 10 años a partir de los 50 años, el riesgo aumenta en $\sim 13 \%{ }^{11}$. También se han descrito otros factores de riesgo como la obesidad, género femenino, diabetes mellitus, hipertensión, enfermedad pulmonar obstructiva crónica, enfermedad renal crónica y síndrome de apnea del sueño. La FAPCC se presenta con mayor frecuencia en procedimientos que afectan al aparato valvular, en particular la válvula mitral, cuando se practica más de un procedimiento de cirugía cardíaca (p. ej., operación valvular más revascularización coronaria), cuando se retiran los BB en el periodo postquirúrgico, cuando la fracción de expulsión del ventrículo izquierdo (FEVI) es menor de $40 \%$ o el diámetro de la aurícula izquierda es mayor de $4 \mathrm{~cm}$, cuando se requiere ventilación mecánica por más de 24 horas, y cuando hay elevación de los valores séricos del péptido natriurético cerebral ${ }^{4,5,10-16}$ (Tabla 1).

La identificación de estos factores de riesgo trajo consigo el desarrollo de herramientas para la predicción de FAPCC. Con ellas se busca identificar a los pacientes con alto riesgo de desarrollar FAPCC, que en teoría son los que más se beneficiarían de un tratamiento profiláctico. A continuación se presentan
Tabla 1. Factores de riesgo de la fibrilación auricular en el posquirúrgico de la cirugía cardíaca

\begin{tabular}{|c|c|}
\hline & Variable \\
\hline \multirow[t]{12}{*}{ Preoperatorios } & Edad avanzada* \\
\hline & Hipertensión arterial sistémica \\
\hline & Crecimiento auricular $>40 \mathrm{~mm}$ \\
\hline & Enfermedad pulmonar obstructiva crónica* \\
\hline & $\begin{array}{l}\text { Fracción de expulsión del ventrículo } \\
\text { izquierdo }<40 \%\end{array}$ \\
\hline & Enfermedad arterial coronaria \\
\hline & Enfermedad valvular mitral o tricuspídea* \\
\hline & $\begin{array}{l}\text { Enfermedad renal crónica } \\
\text { (TFG }<30 \mathrm{ml} / \mathrm{min} / 1.72 \mathrm{~m}^{2 *}\end{array}$ \\
\hline & Obesidad \\
\hline & Tabaquismo \\
\hline & Diabetes mellitus \\
\hline & Consumo de alcohol \\
\hline \multirow[t]{3}{*}{ Intraoperatorios } & Operación con intervención en la válvula mitral* \\
\hline & Uso de bomba de circulación extracorpórea \\
\hline & Pericardiotomía \\
\hline \multirow[t]{4}{*}{ Postoperatorio } & Hipovolemia \\
\hline & Hipervolemia \\
\hline & Retiro de fármacos bloqueadores $\beta^{*}$ \\
\hline & $\begin{array}{l}\text { Retiro de fármacos inhibidores de la enzima } \\
\text { convertidora de angiotensina }\end{array}$ \\
\hline
\end{tabular}

*Principales factores de riesgo para FAPCC.

algunas escalas que pueden utilizarse en la predicción de FAPCC.

\section{MSPI (Multicenter Study of Perioperative Ischemia)}

En 2004 se publicó una puntuación de riesgo, basada en 4,500 pacientes provenientes de 70 hospitales que participaron en el Estudio Multicéntrico de Isquemia Perioperatoria. Al utilizar como variables de predicción la edad (el más importante y con mayor puntuación), antecedente de enfermedad pulmonar obstructiva crónica (EPOC), operación valvular, uso o retiro de fármacos $\mathrm{BB}$ e inhibidores de la enzima convertidora de angiotensina (IECA) y consumo de antiinflamatorios no esteroideos (AINE) o complementación con potasio, dividieron a los pacientes en tres grupos 
Tabla 2. Escala de puntuación de riesgo de aparición de FAPCC MSPI

\begin{tabular}{|c|c|}
\hline Variables & Puntuación \\
\hline \multicolumn{2}{|l|}{ Edad (años) } \\
\hline$<30$ & 6 \\
\hline $30-39$ & 12 \\
\hline $40-49$ & 18 \\
\hline $50-59$ & 24 \\
\hline $60-69$ & 30 \\
\hline 70-79 & 36 \\
\hline$\geq 80$ años & 42 \\
\hline \multicolumn{2}{|l|}{ Antecedentes médicos } \\
\hline Fibrilación auricular & 7 \\
\hline $\begin{array}{l}\text { Enfermedad pulmonar obstructiva } \\
\text { crónica }\end{array}$ & 4 \\
\hline Operación valvular & 6 \\
\hline \multicolumn{2}{|l|}{ Retiro de tratamiento } \\
\hline Bloqueadores $\beta$ & 6 \\
\hline $\begin{array}{l}\text { Inhibidores de la enzima } \\
\text { convertidora de angiotensina }\end{array}$ & 5 \\
\hline \multicolumn{2}{|l|}{$\begin{array}{l}\text { Tratamiento prequirúrgico y } \\
\text { postquirúrgico }\end{array}$} \\
\hline Bloqueadores $\beta$ & -7 \\
\hline $\begin{array}{l}\text { Inhibidores de la enzima } \\
\text { convertidora de angiotensina }\end{array}$ & -5 \\
\hline \multicolumn{2}{|l|}{ Tratamiento postquirúrgico } \\
\hline Bloqueadores $\beta$ & -11 \\
\hline \multicolumn{2}{|l|}{ Otros tratamientos } \\
\hline Complementación de potasio & -5 \\
\hline Antiinflamatorios no esteroideos & -7 \\
\hline
\end{tabular}

(bajo, intermedio y alto). El área bajo la curva (AUC, area under the curve) fue de $0.77^{11}$. Esta escala se modificó en 2009 para incluir el uso de estatinas, si bien el AUC no se modificó (0.77) (Tabla 2) ${ }^{17}$.

\section{Atrial fibrillation risk index}

En 2012 se publicó está puntuación basada en 1,300 pacientes sometidos a intervención de revascularización coronaria, cuya AUC fue de 0.68 (Tabla 3) ${ }^{18}$.
Tabla 3. Atrial fibrillation risk index

\begin{tabular}{|l|c|c|}
\hline Variable & Hombres & Mujeres \\
\hline Edad (años) & $>60$ & $>66$ \\
\hline Peso $(\mathrm{kg})$ & $>76$ & $>64$ \\
\hline Estatura $(\mathrm{cm})$ & $>176 \mathrm{~cm}$ & $>168$ \\
\hline Enfermedad vascular periférica & Presente & Presente \\
\hline
\end{tabular}

Tabla 4. Score POAF

\begin{tabular}{|l|c|c|}
\hline Variables & Puntuación & OR (IC 95\%) \\
\hline Edad & & \\
\hline $60-69$ años & 1 & $2.04(1.81-2.31)$ \\
\hline $70-79$ años & 2 & $2.93(2.60-3.30)$ \\
\hline Más de 80 años & 3 & $3.94(3.31-4.69)$ \\
\hline EPOC & 1 & $1.33(1.14-1.56)$ \\
\hline $\begin{array}{l}\text { Diálisis o } \\
\text { TFG < 15 ml/min/1.73 m² }\end{array}$ & 1 & $1.90(1.17-3.10)$ \\
\hline Operación de urgencia & 1 & $1.50(1.19-1.88)$ \\
\hline $\begin{array}{l}\text { Uso de balón de } \\
\text { contrapulsación intraaórtico }\end{array}$ & 1 & $1.90(1.28-2.83)$ \\
\hline $\begin{array}{l}\text { Fracción de } \\
\text { expulsión < 30\% }\end{array}$ & 1 & $1.45(1.18-1.77)$ \\
\hline Operación valvular & 1 & 1.68 (1.55-1.83) \\
\hline
\end{tabular}

EPOC: enfermedad pulmonar obstructiva crónica; TFG: tasa de filtrado glomerular.

\section{Índice POAF (Postoperative Atrial Fibrillation)}

Esta puntuación publicada en 2014 analizó a alrededor de 17,000 pacientes de tres hospitales universitarios en Europa. De manera similar a otras escalas, la edad fue la variable de mayor peso para la predicción de FAPCC y el AUC fue de 0.64. Dos o más puntos, se relacionó con incidencia mayor de $30 \%$ $(\text { Tabla } 4)^{19}$.

\section{CHA2DS2-VASC}

La escala CHA2DS2-VASc, desarrollada de forma original para establecer el riesgo de embolismo anual en pacientes con $\mathrm{FA}$ en el contexto no quirúrgico ${ }^{20}$, se ha estudiado como herramienta de predicción de FAPCC, incluso en pacientes en ritmo sinusal. El punto de corte de CHA2DS-VASc para predecir FAP es de 3 puntos, el cual tiene sensibilidad, especificidad, valor predictivo positivo y negativo de $84 \%, 84 \%, 43.9 \%$ y $97 \%$, respectivamente. El área bajo la curva es de $0.87^{21}$. 


\section{Comparación de escalas}

Mediante una cohorte aproximada de 1,400 pacientes se realizó la validación de tres de las cuatro escalas comentadas con anterioridad (POAF score, AFRisk index y CHA2DS2-VASC) en 1,416 individuos sometidos a una intervención cardíaca (cirugía de revascularización coronaria, cirugía valvular o procedimientos combinados). No se encontraron diferencias significativas en la capacidad de predecir FAPCC. El POAF score y el CHA2DS2-VASc se relacionaron con inicio del tratamiento profiláctico más frecuente en comparación con aquellos que no usaron alguna escala de riesgo ${ }^{22}$. Sin embargo, cuando se incluyó el índice publicado por Mathew, que agregó como factor adicional el uso de estatinas como factor protector ${ }^{11,17}$, éste se mostró superior para predecir FAPCC. Además, la puntuación CHA2DS2-VASc tuvo un AUC inferior a lo informado en las publicaciones previas ${ }^{17}$, lo cual podría explicarse por las diferencias en la prevalencia de FAPCC de las poblaciones incluidas en las cohortes de validación.

En opinión de los autores, la edad mayor de 60 años, la EPOC, la disfunción ventricular izquierda $(\mathrm{FEVI}<40 \%)$, la enfermedad renal crónica y la operación valvular, sobre todo la que incluye intervención sobre la válvula mitral, son los principales factores relacionados con FAPCC, incluidos en la mayoría de las escalas de riesgo. Ante la presencia de dos o más de estas variables se debe clasificar al paciente en una categoría de riesgo alto y por ende se le debe ofrecer alguno de los tratamientos profilácticos descritos a continuación.

\section{Tratamiento profiláctico}

La relación de la aparición de FAPCC con desenlaces negativos ha llevado a la investigación de intervenciones farmacológicas y no farmacológicas con el objetivo de disminuir su aparición. Un metaanálisis de unos 14,000 pacientes demostró que el tratamiento profiláctico reduce el riesgo de FA en este grupo de pacientes hasta en $32.3 \%^{23}$. Sin embargo, como el tratamiento profiláctico no está exento de efectos secundarios y no se ha establecido con claridad en las principales guías de tratamiento qué grupo de pacientes obtiene beneficio, la indicación de cada intervención debe individualizarse según sea el contexto clínico ${ }^{24}$. A continuación se analizan los agentes utilizados en la profilaxis de la FAPCC.

1. Agentes que actúan de forma predominante al antagonizar el tono adrenérgico. Los bloqueadores adrenérgicos $\beta$ o bloqueadores $\beta$ (BB) son los fármacos más estudiados como tratamiento profiláctico de las arritmias supraventriculares posquirúrgicas, incluido la $\mathrm{FA}^{10}$. El uso de BB reduce la FAPCC de $31.7 \%$ a $16.3 \%$ (OR, 9.33; IC 95\%, 0.26-0.43) $)^{23}$. Sin embargo, el uso de BB no reduce la aparición de episodios vasculares cerebrales o infarto agudo de miocardio ni la mortalidad ${ }^{25}$. Por cada siete pacientes tratados con BB en el periodo perioperatorio se previene un episodio de FAPCC (número necesario a tratar de 7 ) $^{26}$. En la actualidad, el metoprolol es el fármaco más estudiado en la prevención de FAPCC. La dosis de metoprolol es de 50 a $200 \mathrm{mg}$ al día, de acuerdo con el estado hemodinámico, y debe administrarse por lo menos 48 a 72 horas antes del procedimiento quirúrgico. La mayoría de los pacientes sometidos a procedimientos quirúrgicos cardiovasculares utiliza los BB por alguna indicación relacionada con la enfermedad subyacente. Las guías de tratamiento de FA, en el apartado de prevención de FAPCC, sugieren la administración de BB, si no se utilizaban ya (clase I, nivel de evidencia $B)^{20}$. Desde el punto de vista de los autores, los BB son el tratamiento profiláctico de primera línea, sobre todo en los pacientes considerados de alto riesgo. Además, debe evitarse su suspensión durante el periodo postoperatorio, ya que se relacionan con aparición de FAPCC.

\section{Antiarrítmicos}

\section{Amiodarona}

Además del efecto antiarrítmico por el bloqueo de múltiples canales iónicos, la amiodarona tiene efectos simpaticolíticos al bloquear a los receptores adrenérgi$\cos \alpha$ y $\beta$, por lo que también previene la FAPCC. La amiodarona reduce la FAPCC en $57 \%$ al compararse contra el placebo (OR, 0.43; IC 95\%, 0.34-0.54), lo cual se traduce en un número necesario a tratar de $7^{23}$. La mayor parte de los estudios incluidos en el metaanálisis de efectividad de la amiodarona como tratamiento profiláctico incluyó a pacientes tratados previamente con BB, por lo que la contribución real de la amiodarona en la prevención de la FAPCC puede ser menor. La dosis sugerida de amiodarona oral (10 mg/kg/día) debe administrarse seis días antes y seis después de la operación para alcanzar valores tisulares adecuados, lo cual puede limitar su uso en situaciones de urgencia ${ }^{27}$. Una conducta que demostró ser útil en un grupo reducido de pacientes del Instituto Nacional de Cardiología consistió en la administración de 200 mg tres veces al día, por lo menos tres días antes de la 
intervención, $200 \mathrm{mg}$ dos veces al día por 24 horas después de la operación y 200 mg una vez al día hasta el día del alta hospitalaria ${ }^{28}$. Se debe conceder atención a los efectos secundarios como hipotensión (10$30 \%)$, bloqueo auriculoventricular (2\%) y prolongación del intervalo QT corregido (QTc), este último vinculado con arritmias ventriculares $(<1 \%)$; todos ellos pueden requerir la reducción de la dosis o la suspensión del fármaco, hasta en el $11.4 \%$ de los pacientes ${ }^{29}$. Por lo anterior, la guía europea sugiere que la amiodarona sea un esquema profiláctico de la FAPCC (clase II, nivel de evidencia A) y debe considerarse en los pacientes con contraindicaciones para el tratamiento con $\mathrm{BB}^{20}$. Los autores sugieren que, en caso de considerarla como alternativa profiláctica, se emplee el esquema oral descrito con anterioridad, hasta obtener información que permita modificar esta práctica.

\section{Sotalol}

El sotalol, un BB con propiedades de antiarrítmico clase III, es útil para la prevención de la FAPCC. En un metaanálisis que incluyó a 2,988 pacientes redujo la FAPCC de $26.2 \%$ a $16.5 \%$ (OR, 0.55; IC 95\%, $0.41-0.73)^{23}$. Los pacientes que recibieron sotalol no se estratificaron para riesgo de FAPCC y eran más susceptibles de abandonar el tratamiento, en particular por los efectos secundarios como hipotensión y bradicardia; además, se relaciona con arritmias ventriculares como taquicardia helicoidal (torsades de pointes) en 1 a $5 \%$ de los casos $^{3}$. La guía ACC/AHA/HRS de 2014 recomienda usarlo de manera profiláctica (clase II, recomendación B); no obstante, en la guía europea no se prevé su uso ${ }^{26}$. En la opinión de los autores, el sotalol sólo debe considerarse en individuos con contraindicación para el metoprolol y la amiodarona, aunque debe señalarse que no está disponible en México.

\section{Fármacos y sustancias que actúan de modo predominante por sus efectos antiinflamatorios}

\section{Colchicina}

Aunque la colchicina tiene propiedades simpaticolíticas y antiinflamatorias, con un papel potencial en la prevención de la FAPCC, la evidencia es inconsistente. Un subanálisis del estudio Colchicine for the Prevention of the Post-pericardiotomy Syndrome (COPPS) demostró una reducción de $22 \%$ a $12 \%$ en la aparición de FAPCC $^{30,31}$. Una de las principales limitantes de dicha investigación fue el número reducido de pacientes (169 sujetos por grupo), además de que la aparición de FAPCC no fue parte de los desenlaces primarios. Por esta razón se llevó a cabo el estudio COPPS II, cuyo objetivo primario fue valorar la eficacia y seguridad de la colchicina en la prevención de FAP y otras complicaciones posteriores a la pericardiotomía. La colchicina redujo en $7.8 \%$ los casos de FAP; sin embargo, la diferencia no fue estadísticamente significativa. Además, en el grupo de tratamiento con colchicina fueron más frecuentes los efectos adversos gastrointestinales ${ }^{32}$. Una de las posibles diferencias entre el COPPS y el COPPS II es la mayor proporción de procedimientos valvulares en el COPPS II, menos pacientes con operación de revascularización y el hecho de que hasta $20 \%$ de los individuos descontinuó el tratamiento médico. En 2017 se publicó un metaanálisis que incluyó a 1,400 pacientes de cinco estudios clínicos (incluidos COPPS I y COPPS II), con el objetivo de establecer el beneficio de la colqchicina en la profilaxis de FAPCC. El esquema terapéutico empleado en todos los estudios fue de $0.5 \mathrm{mg}$ cada 12 horas. La prevalencia de FAPCC fue de $27 \%$ en el grupo control en comparación con $18 \%$ en el grupo de tratamiento con colchicina (RR, 0.69; IC 95\%, 0.57-0.84). El número necesario a tratar fue de 11. Además, se acompañó de reducción de un día de estancia intrahospitalaria. Los efectos gastrointestinales se incrementaron 2.5 veces en el grupo de tratamiento con colchicina. En apariencia, el principal beneficio del tratamiento con colchicina se encuentra en los pacientes sometidos a operación de revascularización coronaria ${ }^{33}$. A pesar de que la conclusión de este metaanálisis fue que la colchicina tiene beneficio en la reducción de FAPCC, existen otros análisis que no son consistentes con este resultado. En 2018 se publicó una revisión de siete estudios en la que no se identificaron diferencias respecto de la prevención de la FAPCC ${ }^{34}$. Se sugiere el uso de la colchicina en el periodo posquirúrgico con el objetivo de prevenir la aparición de FAPCC, según la guía americana (clase $\mathrm{II}$, recomendación grado $\mathrm{B})^{26}$. Con la evidencia presentada hasta el momento, el papel de la colchicina aún debe demostrarse con otros estudios clínicos de mayor tamaño. Además, debe definirse si la reducción de la dosis puede atenuar los efectos gastrointestinales sin perder efectividad en términos de la prevención de la FAPCC. Podría considerarse la administración de colchicina sólo en los pacientes sometidos a intervención de revascularización coronaria, en quienes el tratamiento con BB y amiodarona está contraindicado. 


\section{Glucocorticoides}

Las concentraciones elevadas de la proteína $C$ reactiva (PCR), como un marcador de inflamación, se relacionan con la aparición de FAPCC y el uso de glucocorticoides reduce su aparición ${ }^{35}$. En 2007 se condujo un estudio clínico asignado al azar que incluyó a 241 pacientes sometidos a revascularización coronaria, cambio valvular aórtico, o ambos, los cuales recibieron $100 \mathrm{mg}$ de hidrocortisona a partir del día de la operación y luego cada 8 horas hasta completar tres días de tratamiento. Se demostró que el esteroide redujo $18 \%$ la FAPCC (OR, 0.54; IC 95\%, 0.35-0.83) con número necesario a tratar de 6 pacientes. Además de recibir esteroide intravenoso, los pacientes se encontraban bajo tratamiento con $\mathrm{BB}$ antes del procedimiento quirúrgico ${ }^{36}$. No obstante, en otro estudio clínico, en el que se incluyó a unos 4,500 pacientes, la dexametasona no redujo la aparición de $\mathrm{FAPCC}^{37}$. A pesar de que existen metaanálisis publicados antes del 2015, en los cuales los esteroides reducen la FAP e incluso los días de estancia intrahospitalaria ${ }^{38,40}$, en 2018 se publicó otro metaanálisis que incluyó a unos 16,000 pacientes, cuyo objetivo fue dilucidar el papel de los esteroides en los pacientes sometidos a operación cardíaca con uso de circulación extracorpórea. No se identificó reducción de la mortalidad entre ambos grupos (OR, 0.85; IC 95\%, 0.71-1.01), pero se documentó un aumento de la incidencia de infarto miocárdico (OR, 1.17; IC $95 \%, 1.04-1.31)$ y menor frecuencia de aparición de FAPCC (OR, 0.91; IC 95\%, 0.86-0.96) ${ }^{41}$. Al desglosar todos los estudios se encontró que el beneficio de los esteroides en la reducción de la incidencia de FAPCC provenía de estudios en los que las poblaciones a estudiar eran inferiores a 120 pacientes. En revisiones previas se sugería el uso de esteroides (hidrocortisona o la dosis equivalente de metilprednisolona) para prevenir la FAPCC. Pese a ello, al analizar la evidencia disponible y los posibles efectos secundarios de la medicación (aumento de la incidencia de infecciones mediastínicas e hiperglucemia), los autores consideran que este grupo de fármacos no debe emplearse para el tratamiento profiláctico de la FAPCC.

\section{Estatinas}

Se han propuesto las estatinas como tratamiento profiláctico en la FAPCC. El mecanismo de acción hipotético es la reducción de mediadores inflamatorios a través de la inhibición de la HMG-CoA reductasa y efectos pleiotrópicos como inhibición plaquetaria, vasodilatación y disminución de la actividad de los linfocitos ${ }^{42}$. En 2015 se analizaron 16 estudios clínicos para valorar el efecto de las estatinas en la mortalidad, infarto miocárdico, FAPCC, episodios embólicos y días de estancia intrahospitalaria en pacientes sometidos a operación cardíaca, los cuales no usaban estatinas con anterioridad. Las estatinas redujeron la mortalidad de $3.4 \%$ a $1.8 \%$ (OR, 0.53; IC 95\%, 0.30-0.94), el infarto miocárdico de $8 \%$ a $4.1 \%$ (OR, 0.54; IC 95\%, 0.38$0.76 \%)$, los días de estancia intrahospitalaria en casi un día menos y la FAPCC de $23.7 \%$ a $12.1 \%$ (OR, 0.53; IC 95\%, 0.43-0.66), con necesidad de tratar a 9 pacientes para prevenir un episodio de FAPCC ${ }^{42}$. En 2016 se publicó el estudio Statin Therapy in Cardiac Surgery (STICS), que asignó al azar a 1,920 pacientes programados para revascularización coronaria o cambio valvular aórtico y recibir rosuvastatina $(20 \mathrm{mg})$ ocho días antes y cinco después de la intervención. El objetivo primario fue documentar la aparición de FAPCC o lesión miocárdica, medido por cambios en la cinética de la troponina. Además de que no se hallaron diferencias entre ambos grupos para el objetivo primario, la rosuvastatina se acompañó de mayores episodios de agudización de la función renal $24.7 \%$ en el grupo de estatinas en comparación con el $19.3 \%$ en el grupo control $\left.^{43}\right)$. En 2018 se llevó a cabo un análisis sobre el efecto de las estatinas en pacientes sometidos a procedimiento cardíaco y no cardíaco de manera electiva. Se incluyó a casi 82,000 pacientes. Las estatinas tuvieron efecto protector contra el infarto miocárdico en pacientes con operación no cardíaca (2.3\% en el grupo de estatinas vs. $5.1 \%$ en el grupo control; OR, 0.44; IC $95 \%, 0.30-0.64)$ con número necesario a tratar de 35. Sin embargo, no hubo efecto en la mortalidad, infarto miocárdico o episodios embólicos en el grupo de intervención cardíaca ${ }^{44}$. Los autores sugieren que el consumo de estatinas se considere parte del tratamiento profiláctico de la FAPCC, en caso de contraindicación para tratamientos con mayor efectividad, sobre todo en procedimientos de revascularización coronaria y cambio valvular aórtico.

\section{Ácidos grasos omega 3}

Los ácidos grasos omega 3 (AGO-3) son ácidos grasos de cadena larga poliinsaturados, que incluyen al ácido eicopentaenoico (EPA) y el docosahexaenoico $(\mathrm{DHA})$, obtenidos principalmente del pescado ${ }^{45}$. Existe evidencia sobre la utilidad de la complementación con AGO-3 en la mortalidad cardiovascular, la isquemia miocárdica y la FAPCC. El mecanismo propuesto es la 
reducción de la inflamación, efectos antioxidantes y disminución del remodelado auricular. En 2002 se publicaron los resultados del estudio OPERA, que asignó al azar a casi 1,500 pacientes para recibir 8 a $10 \mathrm{~g}$ de AGO-3 en el preoperatorio y $2 \mathrm{~g}$ al día en el postoperatorio. El desenlace primario fue la FAPCC con duración mayor de $30 \mathrm{~s}$ y el desenlace secundario fue FAPCC con duración mayor de $1 \mathrm{~h}$, sintomática o con necesidad de tratamiento con cardioversión. Los AGO3 no redujeron la aparición de FA ni se acompañaron de efectos adversos. En este estudio no se midieron las concentraciones séricas de los ácidos grasos ${ }^{46}$. En 2018 se publicaron los resultados de una revisión sistémica sobre los efectos de los AGO-3 sobre la FAPCC. La dosis de AGO-3 entre los estudios incluidos fue variable, $0.5 \mathrm{mg}$ por kilogramo de peso (máximo $100 \mathrm{mg}$ ) intravenoso o 2 a $15 \mathrm{~g}$ vía oral cada 24 horas. Los AGO-3 redujeron la incidencia de FAPCC de $35.7 \%$ a 31.85 ( $p=0.01$; OR, 0.89; IC 95\%, 0.81-0.97). En el análisis de subgrupos, la incidencia de FAP fue menor en los pacientes que recibieron AGO-3 cuya composición EPA/DHA fue menor de 1 y en los pacientes con procedimiento de revascularización. El número necesario a tratar fue de $25^{45}$. Desde el punto de vista de los autores, los AGO-3 forman parte del tratamiento profiláctico de tercera línea para la prevención de FAPCC. En caso de considerar su uso, debe reservarse para pacientes sometidos a revascularización coronaria y con AGO-3 cuya composición EPA/DHA sea menor de 1.

\section{Magnesio}

La hipomagnesemia se vincula con la aparición de FA en el periodo posquirúrgico de un procedimiento cardíaco y el uso de diuréticos y la circulación extracorpórea se acompañan de hipomagnesemia ${ }^{47}$. Un metaanálisis que incluyó a 2,988 pacientes demostró que la prevalencia de FAPCC en el grupo control fue de $26.2 \%$ en comparación con el $16.5 \%$ en el grupo tratado con magnesio (OR, 0.55; IC 95\%, 0.41-0.73\%). En el $57 \%$ de los estudios incluidos, el magnesio se administró por primera vez en el momento de la intervención a una dosis 40 a $80 \mathrm{mg} / \mathrm{kg}^{23}$. Cabe destacar que los estudios incluidos en el metaanálisis excluyeron a pacientes con factores de riesgo conocidos para FAPCC, como edad avanzada, revascularización coronaria o valvular previa y disfunción ventricular. Además, no se ha encontrado una relación consistente entre los valores séricos de magnesio y FAPCC ${ }^{26}$. Las guías canadienses de FA sugieren el uso de magnesio en procedimientos cardíacos, en caso de que el contexto clínico del paciente contraindique el empleo de fármacos con efectividad probada como BB y amiodaro$n^{23,26,47}$. Por el momento no hay evidencia suficiente para sugerir el uso de la complementación con magnesio como medida farmacológica aislada, pero puede considerarse en pacientes de alto riesgo, junto con fármacos como los BB y amiodarona, y en las situaciones con hipomagnesemia documentada.

\section{Ivabradina}

Se ha estudiado como potencial agente para reducir la FAPCC. En 2016 se publicó un estudio clínico asignado al azar, cuyo objetivo primario era comparar la eficacia de la ivabradina en la prevención de FAPCC (iniciado 48 horas antes de la operación y mantenido por una semana después de ella). Se compararon tres esquemas: grupo 1, ivabradina de $5 \mathrm{mg}$ cada 12 horas las primeras 24 horas y luego $7.5 \mathrm{mg}$ cada 12 horas; grupo 2, bisoprolol de $5 \mathrm{mg}$ cada 12 horas; y grupo 3, ivabradina y bisoprolol a las dosis comentadas. La combinación de ivabradina y bisoprolol redujo de manera estadísticamente significativa la FAPCC (4.2\%) en comparación con ivabradina (15.1\%) y bisoprolol (12.2\%) usados de manera aislada ${ }^{48}$. El mecanismo propuesto es secundario al mecanismo de acción de la ivabradina sobre los canales If, cuya densidad parece ser mayor en los miocardiocitos de las venas pulmonares, las cuales son la causa de fibrilación auricular en el 70\% de los $\operatorname{casos}^{49}$. Se necesitan más estudios sobre la combinación de ivabradina y BB para recomendarla como una alternativa útil en la prevención de FAPCC.

\section{Maniobras intervencionistas}

\section{Estimulación auricular}

Las extrasístoles auriculares producen dispersión en el periodo refractario auricular, lo que favorece la aparición de circuitos de reentrada y da lugar a la aparición de FAPCC. Por esta razón se ha propuesto que la estimulación de una o ambas aurículas podría prevenir los efectos electrofisiológicos relacionados con la FAPCC y así disminuir su aparición ${ }^{50}$. Un metaanálisis demostró que la estimulación auricular redujo la aparición de FAPCC de $32.8 \%$ a $18.7 \%$ (OR, 0.47; IC 95\%, 0.36-0.61). La estimulación auricular se realizó mediante diferentes abordajes, como biauricular, auricular izquierdo o derecho y del haz de Bachmann. Sólo cuatro estudios de los 21 incluidos en el metaanálisis 
demostraron beneficio con esta técnica ${ }^{23}$. A pesar de que las guías americanas de tratamiento de la FA sugieren la estimulación auricular para evitar la aparición de FAPCC (recomendación clase $\mathrm{Ilb})^{26}$, los autores consideran que debido a la colocación de electrodos de marcapasos auriculares que no es sistemática, la complejidad técnica de alguno de estos abordajes (haz de Bachmann) y el escaso poder estadístico de los estudios clínicos evaluados con anterioridad, la estimulación auricular no debe formar parte de la profilaxis para la prevención de FAPCC.

\section{Pericardiotomía}

Existen informes aislados de que la pericardiotomía izquierda posterior reduce la incidencia de FAPCC, ya que permite el drenaje en el lado izquierdo del tórax y disminuye la acumulación de sangre en la aurícula izquierda, la cual podría desencadenar FA por inflamación de los miocardiocitos ${ }^{51}$. En la actualidad se realiza la inscripción de pacientes para incluirlos en el Posterior Left Pericardiotomy for the Prevention of Postoperative Atrial Fibrillation after Cardiac Surgery Trial (PALACS), cuyo objetivo es valorar el efecto de la pericardiotomía posterior en la prevención de FAPCC. Además, se valorarán como objetivos secundarios el tiempo total en FAPCC, el tiempo de hospitalización, la mortalidad y los efectos adversos ${ }^{52}$.

\section{Consideraciones sobre la profilaxis de FAPCC}

El tratamiento profiláctico reduce la incidencia de FAPCC; sin embargo, puede acompañarse de efectos secundarios, que en el caso de los bloqueadores $\beta$ corresponden a hipotensión y bloqueo auriculoventricular $y$, en el de los antiarrítmicos de clase III como la amiodarona, a predisposición a arritmias letales como taquicardia o fibrilación ventricular. Por lo anterior, los autores consideran que una medida que puede utilizarse es un uso escalonado (administrar siempre los fármacos con mayor nivel de evidencia) y razonado, reservado para los pacientes considerados de alto riesgo de FAPCC, por ejemplo aquéllos con dos o más factores de riesgo ${ }^{24}$.

\section{Tratamiento de la FAPCC: ¿control del ritmo o de la frecuencia?}

La necesidad de tratamiento, ya sea antiarrítmico o control de la frecuencia, es menor debido a que los episodios son autolimitados y la recurrencia no se presenta en todos los $\operatorname{casos}^{4}$. El tratamiento del episodio agudo debe incluir un abordaje integral para tratar causas potencialmente corregibles como hipoxemia y alteraciones hidroelectrolíticas (en especial potasio y magnesio). La FAPCC puede tratarse mediante dos medidas: control de la frecuencia ventricular y control del ritmo (restauración del ritmo sinusal). La conducta de control del ritmo no muestra diferencias en mortalidad en comparación con el control de la frecuencia, pero se relaciona con mayor incidencia de efectos adversos, derivados del uso de fármacos antiarrítmicos, y mayor número de hospitalizaciones en el seguimiento, junto con los efectos secundarios del tratamiento ${ }^{53}$. En 2016 se publicó un estudio clínico cuyo objetivo principal fue evaluar el efecto del tratamiento del ritmo o de la frecuencia en pacientes con FAPCC. El desenlace primario fue número de días totales en el hospital (incluidas las visitas a urgencias) en los primeros 60 días posterior al inicio de tratamiento. Entre los desenlaces secundarios se encuentran muerte por todas las causas, implante de marcapasos definitivo y efectos adversos de la medicación. No se observaron diferencias entre ambos grupos de tratamiento ${ }^{54}$. Derivado de la información de este estudio clínico, los autores sugieren que el control de la frecuencia ventricular sea la primera línea de tratamiento en pacientes con FAPCC y estabilidad hemodinámica.

\section{Control de la frecuencia ventricular}

El consumo de fármacos con actividad de bloqueo de la conducción del nodo auriculoventricular debe indicarse como tratamiento de primera línea en pacientes con FAPCC sin datos de inestabilidad hemodinámica, como choque cardiogénico, insuficiencia cardíaca o signos de hipoperfusión cerebral, renal o coronaria ${ }^{4,13,26}$. Se sugiere que el objetivo de frecuencia se encuentre entre los 80 y 110 latidos por minuto ${ }^{16}$; sin embargo, la evidencia que sustenta esta recomendación es escasa y proviene de estudios clínicos de pacientes fuera del contexto quirúrgico y con fracción de expulsión preservada ${ }^{53,55}$. De manera similar al tratamiento profiláctico, los fármacos con mejor perfil de eficacia y seguridad son los bloqueadores $\beta$, de preferencia los de acción corta como el esmolol. Están contraindicados en pacientes con asma no tratada, bloqueo de la conducción AV (duración del segmento PR mayor de $240 \mathrm{~ms}$, bloqueo AV de segundo o tercer grados) e insuficiencia cardíaca aguda sintomática. Las guías estadounidenses, europeas y canadienses recomiendan el uso de bloqueadores $\beta$ con niveles de evidencia elevados (clase I, recomendación 
grado $A)^{26}$. En caso de requerir tratamiento adicional, la segunda línea son los antagonistas del calcio no dihidropiridínicos como el verapamil y el diltiazem; no obstante, debido a que tienen acciones de depresión de la contractilidad miocárdica y suelen tener mayor actividad para disminuir la frecuencia ventricular, se debe tener precaución en los pacientes con factores de riesgo para bradicardia. Las contraindicaciones son similares a las de los bloqueadores $\beta$, excepto porque está permitido su uso en pacientes con neumopatía activa. La digoxina tiene propiedades simpaticolíticas, por lo que podría ser útil para lentificar la frecuencia ventricular en pacientes con FAPCC, si bien su uso no se ha estudiado en este contexto ${ }^{10,26}$.

\section{Control del ritmo}

El tratamiento para restablecer el ritmo sinusal, conocido como forma de controlar el ritmo, debe considerarse en los pacientes en quienes a pesar del tratamiento bloqueador $\beta$ o antagonista del calcio no se logra reducir la frecuencia ventricular, y en sujetos con signos de inestabilidad hemodinámica ${ }^{4,10,13,20,26}$. Esta conducta puede llevarse a cabo por medio de la cardioversión farmacológica o eléctrica.

La cardioversión farmacológica en FAPCC incluye el uso de fármacos bloqueadores de los canales iónicos de clase IA, IC o III de la clasificación de Vaughman-Williams. No existen diferencias en eficacia entre las diferentes clases de antiarrítmicos y el perfil de seguridad de cada fármaco es el que determina el fármaco a utilizar. Los antiarrítmicos de clase IC están contraindicados en pacientes con cardiopatía estructural, lo cual limita su uso en los pacientes sometidos a operación cardíaca. La eficacia informada en cada agente para la recuperación a ritmo sinusal es la siguiente: quinidina (64\%), procainamida (61-87\%), amiodarona (41-93\%), sotalol (35$85 \%)$, ibutilida (57\%). En los pacientes en quienes se decidió optar por tratamiento de control del ritmo y que persistan en fibrilación auricular más de 24 horas se sugiere considerar la cardioversión eléctrica ${ }^{4}$. La mayoría de las recomendaciones respecto del tratamiento recomendadas con anterioridad se deriva de las guías de tratamiento de FA no quirúrgica, ya que la evidencia que sustenta su uso en el contexto quirúrgico es pobre.

\section{Tratamiento anticoagulante en pacientes con FAPCC}

La bomba de circulación extracorpórea, el pinzamiento aórtico y la fibrilación auricular se relacionan con embolias sistémicas y el más importante de éstos es el episodio vascular cerebral. En fecha reciente, en el registro EXCEL (Evaluation of XIENCE versus Coronary Artery Bypass Surgery for Effective Revascularization) se encontró que la FAP en pacientes sometidos a revascularización se vincula con la aparición de episodios vasculares cerebrales hasta tres años después de la intervención (6.6\%; HR, 4.19; IC 95\%, 1.74-10.11) y a mayores tasas de mortalidad (HR, 1.8; IC 95\%, 1.22-2.64) ${ }^{56}$. La incidencia de embolismo en pacientes con FAPCC es de 18.3 episodios por cada 1,000 personas/año y la anticoagulación reduce su aparición (HR, 0.55; IC 95\%, 0.32-0.95) $)^{57}$. Las guías sobre el tratamiento de FAPCC sugieren estratificar el riesgo de embolias ${ }^{2}$; los principales factores de riesgo descritos son antecedente de embolismo (ataque isquémico transitorio o episodio vascular cerebral documentado), edad mayor de 75 años, diabetes, insuficiencia cardíaca, hipertensión y enfermedad vascular previa ${ }^{20,26}$. Además del riesgo de embolia sistémica también se debe valorar el riesgo de hemorragia. Las guías europeas de FA sugieren el uso de la escala HASBLED para estratificar el riesgo de hemorragia en los pacientes con $\mathrm{FA}^{20}$. Sin embargo, dicha escala no está validada para los pacientes con FAPCC. La anticoagulación en el periodo postquirúrgico de cirugía cardíaca se relaciona con episodios hemorrágicos mayores, entre ellos el taponamiento cardíaco. La mayor parte de la información sobre riesgo de embolias sistémicas y riesgo de sangrado en pacientes con FAPCC deriva de cohortes y registros de FA crónica no quirúrgica. La información sobre FAPCC es escasa y los estudios clínicos sobre prevención de episodios vasculares cerebrales son aislados.

Debido a la naturaleza transitoria de la FAPCC, las guías de tratamiento propuestas por la AATS (American Associaton of Thoracic Surgeons) sugiere la anticoagulación en caso de que el episodio de FAPCC tenga duración mayor de 48 horas o en caso de considerar la cardioversión eléctrica o farmacológica ${ }^{2}$. La duración del tratamiento anticoagulante no está bien definida, pero en caso de recuperar ritmo sinusal, se sugiere mantener la anticoagulación hasta por cuatro semanas más $2,20,26$. Los fármacos indicados para la anticoagulación son los antagonistas de la vitamina $\mathrm{K}$ (acenocumarol o warfarina) y los anticoagulantes directos, como el dabigatrán, apixabán y rivaroxabán. No existen estudios clínicos sobre el uso de anticoagulantes directos en pacientes con FAP, pero la AATS sugiere su uso (clase II, recomendación grado $A)^{2}$. 


\section{Conclusiones}

La FA es la arritmia más frecuente en los pacientes sometidos a operación cardiovascular y tiene clara relación con desenlaces negativos. El tratamiento profiláctico con BB y amiodarona, sobre todo en pacientes de alto riesgo, se acompaña de disminución de su aparición. Dada su naturaleza transitoria, se sugiere que el tratamiento inicial de FAPCC sea la conducta de control de la frecuencia cardíaca. Se desconoce cuál debe ser la forma de tratamiento de largo plazo, dado que sólo una minoría de pacientes se mantendrá con FA más allá de tres a seis meses. Existen tratamientos novedosos para FAPCC como el tratamiento prequirúrgico en pacientes de alto riesgo con fármacos nuevos como la ivabradina con o sin uso de BB pero se necesita mayor evidencia para sugerir su uso de manera sistemática. Los autores sugieren que los hospitales con capacidad de realizar operaciones cardiovasculares cuenten con registros y estudios prospectivos que generen los conocimientos que permitan eliminar las lagunas que aún existen en este importante campo de la cardiología.

\section{Financiamiento}

Los autores no recibieron patrocinio para llevar a cabo este documento.

\section{Conflicto de intereses}

Los autores declaran no tener ningún conflicto de intereses.

\section{Responsabilidades éticas}

Protección de personas y animales. Los autores declaran que para esta investigación no se han realizado experimentos en seres humanos ni en animales.

Confidencialidad de los datos. Los autores declaran que en este artículo no aparecen datos de pacientes.

Derecho a la privacidad y consentimiento informado. Los autores declaran que en este artículo no aparecen datos de pacientes.

\section{Bibliografía}

1. LaPar DJ, Speir AM, Crosby IK, Fonner E, Brown M, Rich JB, et al. Postoperative atrial fibrillation significantly increases mortality, hospital readmission, and hospital costs. Ann Thorac Surg. 2014;98(2):527-33.

2. Frendl G, Sodickson AC, Chung MK, Waldo AL, Gersh BJ, Tisdale JE, et al. 2014 AATS guidelines for the prevention and management of perioperative atrial fibrillation and flutter for thoracic surgical procedures. J Thorac Cardiovasc Surg. 2014;148(3):153-93.

3. Nair S. Atrial fibrillation after cardiac surgery. Ann Card Anaesth. 2010;13(3):196.

4. Peretto G, Durante A, Limite LR, Cianflone D. Postoperative arrhythmias after cardiac surgery: incidence, risk factors, and therapeutic management. Cardiol Res Pract. 2014;2014:1-15.

5. Filardo G, Damiano RJ, Ailawadi G, Thourani VH, Pollock BD, Sass DM, et al. Epidemiology of new-onset atrial fibrillation following coronary artery bypass graft surgery. Heart. 2018;104(12):985-92.

6. Enríquez $F$, Jiménez $A$. Taquiarritmias postoperatorias en la cirugía cardíaca del adulto. Profilaxis. Cir Cardiovasc. 2010;17(3):259-74.

7. Ishii Y, Schuessler RB, Gaynor SL, Hames K, Damiano RJ. Postoperative atrial fibrillation: the role of the inflammatory response. J Thorac Cardiovasc Surg. 2017;153(6):1357-65.

8. Zakkar M, Ascione R, James AF, Angelini GD, Suleiman MS. Inflammation, oxidative stress and postoperative atrial fibrillation in cardiac surgery. Pharmacol Ther. 2015;154:13-20.

9. St-Onge S, Perrault LP, Demers P, Boyle EM, Gillinov AM, Cox J, et al. Pericardial blood as a trigger for postoperative atrial fibrillation after cardiac surgery. Ann Thorac Surg. 2018;105(1):321-8.

10. Echahidi N, Pibarot P, O'Hara G, Mathieu P. Mechanisms, prevention, and treatment of atrial fibrillation after cardiac surgery. J Am Coll Cardiol. 2008;51(8):793-801.

11. Mathew JP, Fontes ML, Tudor IC, Ramsay J, Duke P, Mazer CD, et al. A multicenter risk index for atrial fibrillation after cardiac surgery. JAMA. 2004;291(4):1720-1729.

12. Selvi M, Gungor $H$, Zencir $C$, Gulasti S, Eryilmaz U, Akgullu C, et al. A new predictor of atrial fibrillation after coronary artery bypass graft surgery: HATCH score. J Investig Med. 2018;66(3):648-52.

13. Bessissow A, Khan J, Devereaux PJ, Álvarez-García J, Alonso-Coello P. Postoperative atrial fibrillation in non-cardiac and cardiac surgery: an overview. J Thromb Haemost. 2015;13:S304-12.

14. Dave S, Nirgude A, Gujjar P, Sharma R. Incidence and risk factors for development of atrial fibrillation after cardiac surgery under cardiopulmonary bypass. Indian J Anaesth. 2018;62(11):887.

15. Akintoye E, Sellke F, Marchioli R, Tavazzi L, Mozaffarian D. Factors associated with postoperative atrial fibrillation and other adverse events after cardiac surgery. J Thorac Cardiovasc Surg. 2018;155(1):242-251.e10.

16. Lomivorotov VV, Efremov SM, Pokushalov EA, Karaskov AM. New-onset atrial fibrillation after cardiac surgery: pathophysiology, prophylaxis, and treatment. J Cardiothorac Vasc Anesth. 2016;30(1):200-16.

17. Klinger RY, Thunberg CA, White WD, Fontes M, Waldron NH, Piccini JP, et al. Intraoperative magnesium administration does not reduce postoperative atrial fibrillation after cardiac surgery. Anesth Analg. 2015;121(4):861-7.

18. El-Chami MF, Kilgo PD, Elfstrom KM, Halkos M, Thourani V, Lattouf OM, et al. Prediction of new onset atrial fibrillation after cardiac revascularization surgery. Am J Cardiol. 2012;110(5):649-54.

19. Mariscalco G, Biancari F, Zanobini M, Cottini M, Piffaretti G, Saccocci M et al. Bedside tool for predicting the risk of postoperative atrial fibrillation after cardiac surgery: The POAF Score. J Am Heart Assoc. 2014; $3(2): 1-9$.

20. Agewall S, Camm J, Barón Esquivias G, Budts W, Carerj S, Casselman F, et al. Guía ESC 2016 sobre el diagnóstico y tratamiento de la fibrilación auricular, desarrollada en colaboración con la EACTS. Rev Esp Cardiol. 2017;70(1):e1-e84.

21. Borde D, Gandhe U, Hargave N, Mathew M, Joshi S, Pandey K. Prediction of postoperative atrial fibrillation after coronary artery bypass grafting surgery: Is CHA2DS2 -VASc score useful? Ann Card Anaesth. 2014;17(3):182-187.

22. Cameron MJ, Tran DTT, Abboud J, Newton EK, Rashidian H, Dupuis J-Y. Prospective external validation of three preoperative risk scores for prediction of new onset atrial fibrillation after cardiac surgery. Anesth Analg. 2018;126(1):33-8.

23. Arsenault KA, Yusuf AM, Crystal E, Healey JS, Morillo C, Nair GM, et al. Interventions for preventing post-operative atrial fibrillation in patients undergoing heart surgery. Cochrane Database of Systematic Reviews. 2013;1(3611):1-219.

24. Geovanini GR, Alves RJ, de Brito G, Miguel GAS, Glauser VA, Nakiri K. Fibrilación atrial en el postoperatorio de cirugía cardíaca: ¿Quién debe recibir quimioprofilaxia? Arq Bras Cardio. 2009;92(4):316-320.

25. Thein PM, White K, Banker K, Lunny C, Mirzaee S, Nasis A. Preoperative use of oral beta-adrenergic blocking agents and the incidence of new-onset atrial fibrillation after cardiac surgery. A systematic review and meta-analysis. Heart Lung Circ. 2018;27(3):310-21.

26. Ha ACT, Mazer CD, Verma S, Yanagawa B, Verma A. Management of postoperative atrial fibrillation after cardiac surgery. Curr Opin Cardiol. 2016;31(2):183-90.

27. Mitchell LB, Exner DV, Wyse DG, Connolly CJ, Prystai GD, Bayes AJ, et al. Prophylactic oral amiodarone for the prevention of arrhythmias that begin early after revascularization, valve replacement, or repair: PAPABEAR: a randomized controlled trial. JAMA. 2005: 28;294(24): 3093-3100. 


\section{L.A. Baeza-Herrera, et al.: Fibrilación auricular posquirúrgica}

28. Nava-Townsend SRA. Amiodarona oral para fibrilación auricular en pacientes programados a cirugía cardiotorácica [Tesis de Especialidad] Ciudad de México: Universidad Autónoma de México, 2002:13.

29. Raiten J, Patel PA, Gutsche J. Management of postoperative atrial fibriIlation in cardiac surgery patients. Semin Cardiothorac Vasc Anesth. 2015;19(2):122-9.

30. Imazio M, Trinchero R, Brucato A, Rovere ME, Gandino A, Cemin R, et al. COlchicine for the Prevention of the Post-pericardiotomy Syndrome (COPPS): a multicentre, randomized, double-blind, placebo-controlled trial. Eur Heart J. 2010;31(22):2749-54.

31. Imazio M, Brucato A, Ferrazzi P, Rovere ME, Gandino A, Cemin R, et al. Colchicine reduces postoperative atrial fibrillation: results of the Colchicine for the Prevention of the Postpericardiotomy Syndrome (COPPS) atrial fibrillation substudy. Circulation. 2011;124(21):2290-5.

32. Imazio M, Brucato A, Ferrazzi P, Pullara A, Adler $Y$, Barosi A, et al. Colchicine for prevention of postpericardiotomy syndrome and postoperative atrial fibrillation: the COPPS-2 randomized clinical trial. JAMA 2014:312(10):1016-1023.

33. Lennerz C, Barman M, Tantawy M, Sopher M, Whittaker P. Colchicine for primary prevention of atrial fibrillation after open-heart surgery: systematic review and meta-analysis. Int J Cardiol. 2017;249:127-37.

34. Deftereos SG, Vrachatis DA, Angelidis C, Vrettou A-R, Sarri EK, Giota$\mathrm{ki} S \mathrm{G}$, et al. The role of colchicine in treating postoperative and post-catheter ablation atrial fibrillation. Clin Ther. 2019;41(1):21-29.

35. Dernellis J, Panaretou M. Relationship between C-reactive protein concentrations during glucocorticoid therapy and recurrent atrial fibrillation. European Heart Journal. 2004;25:1100-1107.

36. Halonen J, Halonen P, Järvinen O, Taskinen P, Auvinen T, Tarkka M, et al. Corticosteroids for the prevention of atrial fibrillation after cardiac surgery: a randomized controlled trial. JAMA. 2007;297(14):1562-1567.

37. Dieleman JM. Intraoperative high-dose dexamethasone for cardiac surgery: a randomized controlled trial. JAMA. 2012;308(17):1761-1767.

38. Baker WL, White CM, Kluger J, Denowitz A, Konecny CP, Coleman CI. Effect of perioperative corticosteroid use on the incidence of postcardiothoracic surgery atrial fibrillation and length of stay. Heart Rhythm. 2007;4(4):461-468.

39. Marik PE, Fromm R. The efficacy and dosage effect of corticosteroids for the prevention of atrial fibrillation after cardiac surgery: a systematic review. J Crit Care. 2009;(3):458-63.

40. Cappabianca G, Rotunno C, de Luca Tupputi Schinosa L, Ranieri VM Paparella D. Protective effects of steroids in cardiac surgery: a meta-analysis of randomized double-blind trials. J Cardiothorac Vasc Anesth. $201 ; 25(1): 156-65$.

41. Dvirnik N, Belley-Cote EP, Hanif H, Devereaux PJ, Lamy A, Dieleman JM, et al. Steroids in cardiac surgery: a systematic review and meta-analysis. Br J Anaesth. 2018;120(4):657-67.

42. de Waal BA, Buise MP, van Zundert AAJ. Perioperative statin therapy in patients at high risk for cardiovascular morbidity undergoing surgery: a review. Br J Anaesth. 2015;114(1):44-52.
43. Zheng Z, Jayaram R, Jiang L, Emberson J, Zhao Y, Li Q, et al. Perioperative rosuvastatin in cardiac surgery. N Engl J Med. 2016;374(18): 1744-53.

44. Putzu A, de Carvalho e Silva CMPD, de Almeida JP, Belletti A, Cassina $T$, Landoni $G$, et al. Perioperative statin therapy in cardiac and non-cardiac surgery: a systematic review and meta-analysis of randomized controlled trials. Ann Intensive Care. 2018;8(95):1-14.

45. Wang $\mathrm{H}$, Chen J, Zhao L. N-3 polyunsaturated fatty acids for prevention of postoperative atrial fibrillation: updated meta-analysis and systematic review. J Interv Card Electrophysiol. 2018;51(2):105-15.

46. Mozaffarian D. Fish oil and postoperative atrial fibrillation: the omega-3 fatty acids for Prevention of Post-operative Atrial Fibrillation (OPERA) randomized trial. JAMA. 2013;44(3):2000-2011.

47. Jedwab R, Redley B, Hutchinson A. Magnesium sulphate replacement therapy in cardiac surgery patients: a systematic review. Aust Crit Care. 2018;31(2):1-13

48. Abdel-Salam Z, Nammas W. Atrial fibrillation after coronary artery bypass surgery: can ivabradine reduce its occurrence? Ivabradine in postoperative atrial fibrillation. J Cardiovasc Electrophysiol. 2016;27(6):670-6.

49. Chen Y-C, Pan N-H, Cheng C-C, Higa S, Chen Y-J, Chen S-A. Heterogeneous expression of potassium currents and pacemaker currents potentially regulates arrhythmogenesis of pulmonary vein cardiomyocytes. J Cardiovasc Electrophysiol. 2009;20(9):1039-45.

50. Coma Samartín R, de Blas RC, Castaño Ruiz M. Estimulación cardíaca temporal. Estimulación tras cirugía cardíaca. Rev Esp Cardiol. 2007;7(7):54-68

51. Kaleda VI, McCormack DJ, Shipolini AR. Does posterior pericardiotomy reduce the incidence of atrial fibrillation after coronary artery bypass grafting surgery? Interact Cardiovasc Thorac Surg. 2012;14(4):384-9.

52. Abouarab AA, Leonard JR, Ohmes LB, Lau C, Rong LQ, Ivascu NS, et al. Posterior left pericardiotomy for the Prevention of Postoperative Atrial Fibrillation after Cardiac Surgery (PALACS): study protocol for a randomized controlled trial. Trials. 2017;18(593):1-10.

53. Wyse DG, Awaldo AL, DiMarco JP, Domanski MJ, Rosenberg $Y$, Schron EB, et al. A comparison of rate control and rhythm control in patients with atrial fibrillation. N Engl J Med. 2002;347(23):1825-1833.

54. Gillinov AM, Bagiella E, Moskowitz AJ, Raiten JM, Groh MA, Bowdish $M E$, et al. Rate control versus rhythm control for atrial fibrillation after cardiac surgery. N Engl J Med. 2016;374(20):1911-21.

55. Van Gelder IC, Groenveld HF, Crijns HJGM, Tuininga YS, Tijssen JGP, Alings $\mathrm{AM}$, et al. Lenient versus strict rate control in patients with atrial fibrillation. N Engl J Med. 2010;362(15):1363-73.

56. Kosmidou I, Chen S, Kappetein AP, Serruys PW, Gersh BJ, Puskas JD, et al. New-onset atrial fibrillation after PCI or CABG for left main disease. J Am Coll Cardiol. 2018;71(7):739-48.

57. Butt JH, Xian Y, Peterson ED, Olsen PS, Rørth R, Gundlund A, et al. Long-term thromboembolic risk in patients with postoperative atrial fibrillation after coronary artery bypass graft surgery and patients with nonvalvular atrial fibrillation. JAMA Cardiol. 2018;3(5):417. 